\title{
A pilot process to identify and switch high-risk warfarin patients to direct oral anticoagulants
}

\author{
Samson C. Lee ${ }^{1}$, James B. Groce III $^{2,3,4}$, Jennifer J. Kim*4,5,6 \\ ${ }^{1}$ University of California Davis Medical Center, Sacramento, United States \\ ${ }^{2}$ Campbell University School of Pharmacy, Buies Creek, United States \\ ${ }^{3}$ University of North Carolina School of Medicine, Chapel Hill, United States \\ ${ }^{4}$ Moses Cone Memorial Hospital, Greensboro, United States \\ ${ }^{5}$ University of North Carolina Eshelman School of Pharmacy, Chapel Hill, United States \\ ${ }^{6}$ Area Health Education Center, Greensboro, United States
}

Received: July 9, 2017

DOI: $10.5430 /$ jha.v6n5p20
Accepted: September 4, 2017 Online Published: September 18, 2017

URL: https://doi.org/10.5430/jha.v6n5p20

\begin{abstract}
Objective: To pilot a process for identifying and switching high-risk warfarin patients to direct oral anticoagulants (DOACs) in nonvalvular atrial fibrillation (AF) or venous thromboembolism (VTE).

Methods: A pharmacy resident identified high-risk warfarin patients using three criteria. The resident reviewed medical charts, communicated recommendations to the primary care physician (PCP), and scheduled patients for appointments to switch to DOACs. Patients were followed every 1 to 3 months after initiating DOACs. The primary outcome of the study was the percentage of high-risk warfarin patients before and after process implementation. Secondary outcomes at 6 months included bleeding or thrombotic events, patient-reported side effects, recommendation acceptance rates, adherence rates, clinic time in therapeutic range (TTR), and patient satisfaction.

Results: Out of 76 patients evaluated, 22.4\% were identified as high-risk using the pre-specified criteria. After program implementation, this percentage was reduced to $9.2 \%$. No significant adverse events occurred by 3-month follow-up, and $100 \%$ of recommendations were accepted by physicians. Adherence rates and clinic TTR also improved after implementation (87.5\% to $94 \%$ and $60.9 \%$ to $81.1 \%$, respectively). Overall, patients reported satisfaction with switching from warfarin to DOAC.

Conclusions: A pilot process was successful in reducing the percentage of high-risk warfarin patients by switching to DOAC therapy.
\end{abstract}

Key Words: Warfarin, Direct oral anticoagulant, Anticoagulation safety, Quality improvement, Interprofessional care

\section{INTRODUCTION}

Atrial fibrillation (AF) is a common cardiac arrhythmia encountered in clinical practice. AF is associated with a high rate of cardiovascular and cerebrovascular morbidity and mortality resulting in significant healthcare resource utilization and public health burden. ${ }^{[1]}$ The estimated US prevalence of $\mathrm{AF}$ of 5.1 million is predicted to increase to 9.3 million by 2030. ${ }^{[2]}$ Venous thromboembolism (VTE) is the third most common cardiovascular illness after acute coronary syndrome and stroke. The precise number of people affected by VTE is unknown, but the overall annual incidence of VTE in the United States is estimated to be between 1 and 2 per $1,000 \cdot{ }^{[3]}$ Given the high prevalence of these disease states, there is strong need to optimize anticoagulation

\footnotetext{
*Correspondence: Jennifer J. Kim; Email: jen.kim@ conehealth.com; Address: 1200 North Elm Street, Ground Floor, Greensboro, North Carolina, 27401, United States.
} 
therapy because it is the mainstay for the prevention of stroke in the setting of $\mathrm{AF}$ and for the treatment and prevention of VTE.

Discovered in the 1920s and approved for clinical use in the 1950s, anticoagulation with warfarin has been the standard of care for decades. However, optimal anticoagulation with warfarin has remained challenging because of multiple drugdrug and food-drug interactions, genetic variability, and narrow therapeutic index. Warfarin requires regular monitoring and dose adjustments to maintain a therapeutic international normalized ratio (INR). ${ }^{[4]}$

In 2010 the Food and Drug Administration approved dabigatran, a direct oral anticoagulant (DOAC) for the prevention of stroke or systemic embolism in the setting of non-valvular atrial fibrillation (NVAF) which excludes those with mechanical prosthetic heart valves or moderate to severe mitral stenosis. Subsequently, other DOACs have been approved including rivaroxaban, apixaban, and edoxaban. DOACs provide comparable or better efficacy and safety, predictable pharmacokinetics, less drug-drug and drug-food interactions, and no routine therapeutic laboratory monitoring. ${ }^{[5]}$ National guidelines now include DOACs as recommended treatment options along with warfarin. ${ }^{[6,7]}$ Structured follow-up and monitoring is recommended for patients and can be taken on by pharmacists in various settings. ${ }^{[8]}$

Patients on long-term warfarin may be optimally managed and maintained on warfarin; however, options are limited for patients experiencing challenges yet requiring long-term anticoagulation therapy. ${ }^{[9]}$ Some options include increasing the INR target for patients experiencing a thrombotic event while being within the target INR range or switching to another agent such as unfractionated heparin, low molecular weight heparin, aspirin, or a DOAC. To date, there are no identified published reports of a systematic approach to select appropriate patients on warfarin to switch to a DOAC. Time in therapeutic range (TTR) is often used as a quality marker for anticoagulation management for patients maintained on warfarin but its utility may be limited in patients such as newstarts or those experiencing medication changes. ${ }^{[10]}$ The SAMe-TT2R2 is a scoring tool developed for AF patients that may help with selection between warfarin and DOAC therapy. ${ }^{[11]}$ The purpose of this study was to pilot a process for identifying and switching high-risk warfarin patients to DOACs in NVAF or VTE.

\section{MethodS}

Cone Health Internal Medicine Center is a primary care clinic within Moses H. Cone Memorial Hospital that provides care for adult patients regardless of financial status. It is part

Published by Sciedu Press of Cone Health Medical Group and is the teaching site for the Internal Medicine Residency Program. Clinic staff consists of 12 attending and 22 resident physicians, a social worker, financial counselor, dietician, nurses, and clinical pharmacists (approximately 0.6 full time equivalent [FTE] split faculty positions). Payor mix consists of approximately 45\% Medicare, $15 \%$ Medicaid, 15\% commercial, and 10\% self-pay. Charity care comprises about $20 \%$ of the population. The pharmacist-run Anticoagulation Clinic cares for approximately 130 patients and utilizes Dose Response ${ }^{\circledR}$ to monitor INR and TTR via the Rosendaal method.

This was a single-center quality improvement study approved by Cone Health Institutional Review Board. A pharmacy resident used three criteria to categorize warfarin patients as high-risk. First, patients with a suboptimal history of therapeutic INR laboratory values were defined as having a one-year TTR $<60 \%$. The second criterion required a poor history of clinic attendance defined as less than $75 \%$ completed appointments within one year. The third criterion required a SAMe-TT2R2 score greater than 2, indicating a high probability of subtherapeutic warfarin control. ${ }^{[10]}$ Patients who met all three criteria were evaluated by the pharmacy resident for switch to a DOAC.

Eligible patients for the study were at least 18 years of age on warfarin with an active profile within the DoseResponse ${ }^{\circledR}$ system. Patients were excluded if they had a contraindication to a DOAC (i.e. mechanical prosthetic valve) or were already switched to a DOAC prior to patient encounter. The full description of inclusion and exclusion criteria is illustrated in Figure 1. The pharmacy resident reviewed medical charts, communicated recommendations to the primary care physician (PCP), and scheduled patients for appointments to switch to DOACs. The pharmacy resident collaborated with nurses, social worker, financial counselor, or clerical staff as appropriate to coordinate care for each patient. During the initial appointment, INR and other appropriate laboratory parameters (e.g. renal or hepatic function) were obtained. Lastly, patients were provided education regarding their medical condition and the appropriate use of the DOAC. Pharmacy resident follow up appointments occurred 1 month and 3 months after DOAC initiation, and a phone call occurred at 6 months including a patient satisfaction survey. ${ }^{[8]} \mathrm{Pa}-$ tients and pharmacies were also contacted monthly to review adherence.

The primary outcome of the study was percentage of highrisk warfarin patients. Secondary outcomes at 6 months following DOAC initiation included major bleeding events, as defined by the International Society of Thrombosis and Haemostasis (fatal bleeding, and/or symptomatic bleeding 
in a critical organ or area, and/or bleeding causing a fall in hemoglobin level of $\geq 2 \mathrm{~g} / \mathrm{dl}$ or $1.24 \mathrm{mmol} / \mathrm{L}$ leading to $\geq 2$ units of transfused whole blood or red cells), ${ }^{[12]}$ new or recurrent thrombotic events, any patient-reported side effects, and patient satisfaction. Additional analyses included physician acceptance of recommendations, total number of successful patients transitioned during the study period, and overall clinic TTR pre-post intervention. Medication adherence was evaluated by calculating the medication proportion of days covered (PDC) after contacting pharmacies for refill histories.
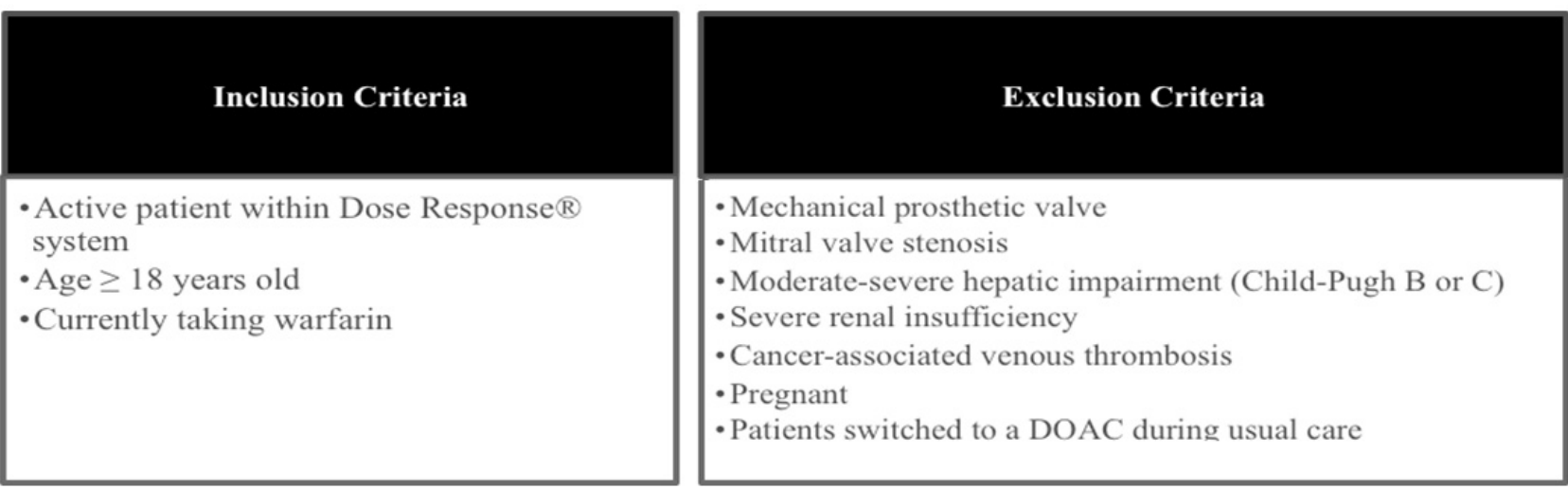

Figure 1. Inclusion and exclusion criteria

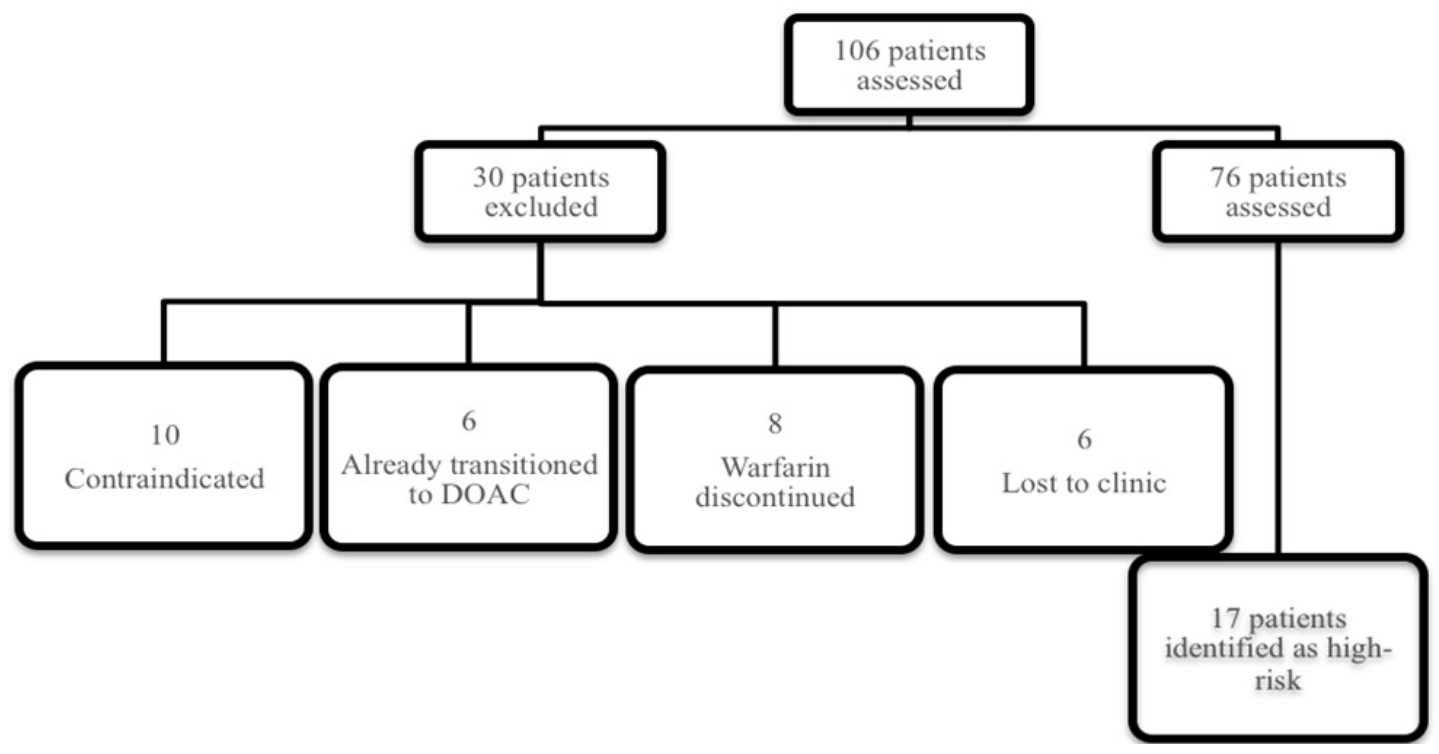

Figure 2. Enrollmen

\section{RESUlts}

A total of 106 clinic patients were identified for screening from the Dose Response ${ }^{\circledR}$ system (see Figure 1). Thirty patients $(28.3 \%)$ were excluded for contraindications to a DOAC (10), currently receiving a DOAC (6), discontinued anticoagulation therapy (8), or being lost to clinic followup (6). Seventy-six patients were evaluated of which 17 $(22.4 \%)$ were identified as high-risk due to meeting all three pre-specified criteria (see Figure 2).
The mean age of the high-risk population was 57.5 years with 2 patients being of Caucasian descent (see Table 1). The most common indication for anticoagulation therapy was VTE, which was consistent with the overall clinic population. The majority of the high-risk patients had concomitant hypertension and tobacco use within the past 2 years, $76.5 \%$ and $70.6 \%$ respectively. In this high-risk group, the mean TTR for was low at $34.6 \%$. Likewise, clinic appointment attendance was poor at $56.7 \%$. The mean SAMe-TT2R2 was 4.8 (range $3-7$ ). 
Table 1. Baseline characteristics

\begin{tabular}{|c|c|c|c|}
\hline Patient Characteristic & Overall Warfarin Population $(\mathrm{N}=76)$ & \multicolumn{2}{|c|}{ High-risk Warfarin Patients $(\mathrm{N}=17)$} \\
\hline Age, years & 60.1 & \multicolumn{2}{|c|}{57.5} \\
\hline - Race - white, no. (\%) & $24(31.6)$ & \multicolumn{2}{|l|}{$2(11.8)$} \\
\hline - Sex - male, , no. $(\%)$ & $38(50.0)$ & \multicolumn{2}{|l|}{$6(35.3)$} \\
\hline - Atrial fibrillation, no. (\%) & $24(31.6)$ & \multicolumn{2}{|l|}{$6(35.3)$} \\
\hline - Venous thromboembolism, no. (\%) & $52(68.4)$ & \multicolumn{2}{|l|}{$11(64.7)$} \\
\hline \multicolumn{4}{|l|}{ Comorbidities } \\
\hline - $\mathrm{CHF}$, no. $(\%)$ & $11(14.5)$ & \multicolumn{2}{|l|}{$3(17.7)$} \\
\hline - Hypertension, no. (\%) & $52(68.4)$ & \multicolumn{2}{|l|}{$13(76.5)$} \\
\hline - Diabetes, no. $(\%)$ & $21(27.6)$ & \multicolumn{2}{|l|}{$8(47.1)$} \\
\hline - Stroke, no. (\%) & $12(15.8)$ & \multicolumn{2}{|l|}{$2(11.8)$} \\
\hline - Tobacco use, no. $(\%)$ & $36(47.4)$ & \multicolumn{2}{|l|}{$12(70.6)$} \\
\hline Appointment attendance $<75 \%$, no. $(\%)$ & $46(60.5)$ & $\begin{array}{l}\text { Mean \% appointment attendance } \\
\text { (Range) }\end{array}$ & $\begin{array}{l}56.7 \% \\
(25-72.5)\end{array}$ \\
\hline TTR < $60 \%$, no. $(\%)$ & $28(36.8)$ & $\begin{array}{l}\text { Mean TTR } \\
\text { (Range) }\end{array}$ & $\begin{array}{l}34.6 \\
(14.1-55)\end{array}$ \\
\hline SAMe-TT2R2 $\leq 2$, no. $(\%)$ & $19(25.0)$ & Mean score & 4.8 \\
\hline SAMe-TT2R2 > 2, no. (\%) & $57(75.0)$ & (Range) & $(3-7)$ \\
\hline
\end{tabular}

Note. Table 1 illustrates baseline characteristics of the population managed. Of note, all high-risk warfarin patients had an appointment attendance $<75 \%$, TTR $<60 \%$, and SAMe-TT2R2 $>2$; therefore Table 1 displays the mean and range for these data points for high-risk warfarin patients

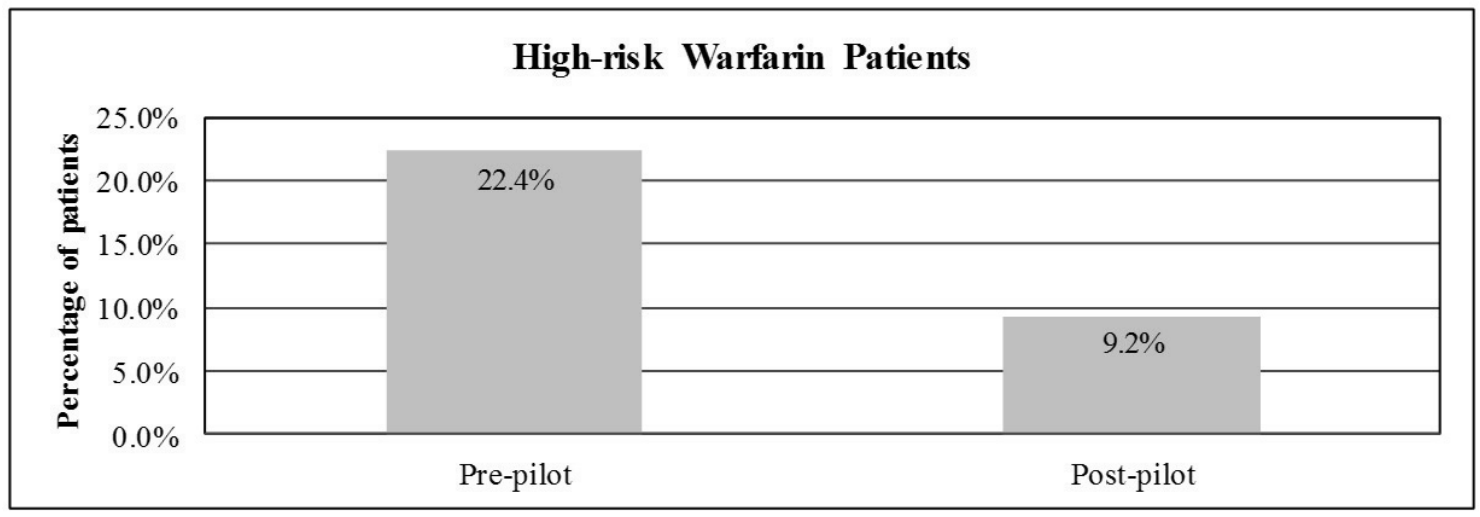

Figure 3. Primary endpoint

Table 2. Secondary endpoints

\begin{tabular}{lll}
\hline Event & High-risk Warfarin Patients & \\
\hline Major bleeding event, no. (\%) & $0(0)$ & \\
Side effects, no. (\%) & $2(20)$ & \\
Thrombotic events, no. (\%) & $0(0)$ & Post-implementation, 81.1 \\
Physician acceptance, no. (\%) & $17(100)$ & After transition to DOAC, 94 \\
Overall Clinic TTR, (\%) & Pre-implementation, 60.9 & Before transition to DOAC, 87.5 \\
Mean PDC, (\%) & & \\
\hline
\end{tabular}

A total of 10 high-risk patients were transitioned to a DOAC (see Figure 3). The percentage of high-risk warfarin patients was reduced by $13.2 \%$ (from $22.4 \%$ to $9.2 \%$ ) after program implementation. No major bleeding events or thrombotic events have been reported since the publication of this article. One patient reported headaches after initiation of rivaroxa- ban, a treatment-emergent adverse event reported in $4.56 \%$ of the treatment arm in the ROCKET-AF study. ${ }^{[13]}$ The side effect resolved in this patient after weeks of continuing rivaroxaban, but other clinical factors including hypoglycemia which the patient also experienced around the same time of rivaroxaban initiation may have contributed. Another pa- 
tient reported nausea but this patient also experienced nausea while on warfarin. This patient was switched to a different DOAC and further follow-up will be provided.

All warfarin-to-DOAC pharmacist recommendations to PCPs were accepted (see Table 2). Medication adherence evaluation using PDC was $60 \%$ prior to the study. After the study, the PDC was 94\%, indicating an improvement in patients transitioned to DOAC. The overall TTR for the clinic also improved from $60.9 \%$ to $81.1 \%$. Most patients reported satisfaction with the switch from warfarin to DOAC, with a median score of 5, indicating very satisfied, for most of the survey items (see Table 3 ).

Table 3. Satisfaction survey of high-risk warfarin patients switched from warfarin to DOAC therapy

\begin{tabular}{|c|c|c|c|c|c|c|}
\hline & $\begin{array}{l}\text { Very dissatisfied } \\
(\text { Score }=1) \\
\text { No., }(\%)\end{array}$ & $\begin{array}{l}\text { Dissatisfied } \\
(\text { Score }=2) \\
\text { No., }(\%)\end{array}$ & $\begin{array}{l}\text { Neutral } \\
(\text { Score }=3) \\
\text { No., }(\%)\end{array}$ & $\begin{array}{l}\text { Satisfied } \\
(\text { Score }=4) \\
\text { No., }(\%)\end{array}$ & $\begin{array}{l}\text { Very Satisfied } \\
(\text { Score }=5) \\
\text { No., }(\%)\end{array}$ & $\begin{array}{l}\text { Median } \\
\text { Score }\end{array}$ \\
\hline Switch from warfarin & 0 & 0 & $1(10)$ & $3(30)$ & $6(60)$ & 5 \\
\hline Lack of laboratory monitoring & 0 & 0 & $1(10)$ & $3(30)$ & $6(60)$ & 5 \\
\hline Lower bleed risk & 0 & 0 & $3(30)$ & $1(10)$ & $6(60)$ & 5 \\
\hline Less food interactions & 0 & 0 & $2(20)$ & $2(20)$ & $6(60)$ & 5 \\
\hline Efficacy & 0 & 0 & $3(30)$ & $2(20)$ & $5(50)$ & 4.5 \\
\hline Dosing & 0 & 0 & $2(20)$ & $1(10)$ & $7(70)$ & 5 \\
\hline Cost & 0 & 0 & $1(10)$ & $1(10)$ & $8(80)$ & 5 \\
\hline Education provided by clinic team & 0 & 0 & 0 & $3(30)$ & $7(70)$ & 5 \\
\hline Follow-up by clinic team & 0 & 0 & 0 & $2(20)$ & $8(80)$ & 5 \\
\hline
\end{tabular}

\section{Discussion}

A pharmacy resident successfully piloted a process to convert high-risk warfarin patients to DOACs with no reports of significant adverse drug events. We used an interprofessional population management strategy to risk stratify patients and implement changes. Medical staff was overall receptive to pharmacist recommendations as illustrated by the acceptance rate. High-risk warfarin patients who were not switched to DOACs were due to patient refusal. A common barrier was patient preference to remain on warfarin, even after education on risks and benefits of warfarin compared to DOAC therapy were provided. This is perhaps due to the prospective laboratory monitoring with warfarin providing closure regarding therapeutic safety and efficacy, in addition to more interaction with the clinical team. ${ }^{[14,15]}$

Cost was another common but not a complete barrier to transitioning to a DOAC. In some situations, DOAC copays were less than the combined cost of warfarin copays, clinic appointment copays and transportation. In other instances, free samples or copay cards were provided to help offset cost. Patients without insurance were enrolled in pharmaceutical manufacturer patient assistance programs (PAPs). The cost strategies utilized in this pilot study have been adopted in our clinic for any patient started on a DOAC. Physicians and clinic staff now re-direct patients with cost barriers to multidisciplinary members including a financial counselor, social worker, or clinical pharmacist for DOAC cost support. Nurses facilitate prior authorizations, but, if unsuccessful, will re-direct patients through this process as well. A future pilot study will be implemented to provide continuous
DOAC monitoring, including re-assessments of financial and insurance status to help ensure patients can be maintained on DOAC therapy.

The three high-risk criteria were selected in order to identify patients most likely to benefit from transitioning to DOACs. Relying solely on TTR may provide an inaccurate assessment of INR control. For instance, new-start warfarin patients are more likely to have labile INRs, leading to a low TTR initially, not necessarily indicating poor candidacy for long-term therapy with warfarin. Also, if an INR is out of range and the time between measurements is long (e.g. due to high no-show rate), the TTR will be falsely low. Other scenarios may include medication, dietary, or acute illness. ${ }^{[10]}$ We added the criteria of appointment adherence and SAMeTT2R2 score to account for potential pitfalls of the TTR. The change in percentage of high-risk warfarin patients may have been greater if only 2 rather than 3 criteria were utilized. We plan to expand our pilot to more patients who may be better suited for DOAC therapy by changing the criteria utilized (e.g. TTR plus SAMe-TT2R2, TTR plus appointment adherence, or appointment adherence plus SAMe-TT2R2) and we encourage exploration of other approaches individualized to each practice setting.

Poor INR history was defined as TTR $<60 \%$ considering the average TTR reported in clinical trials comparing warfarin to DOACs as well as data reporting capabilities specific to the Dose Response ${ }^{\circledR}$ system. Facilities may lack software to calculate TTR which may limit the application of this approach at other institutions. Other methods of calculating TTR that can be used include (1) the proportion of patients 
with a therapeutic INR at a specific point in time or (2) number of therapeutic INRs over total number visits over a period of time, but these methods have been found to overestimate TTR. If TTR is not available, other criteria may be used such as clinic appointment adherence and/or the SAMe-TT2R2 score.

The appointment adherence cut-point of $75 \%$ was adopted from a study of a different population demographic due to a lack of literature on the concept. ${ }^{[16]}$ The SAMe-TT2R2 scoring tool was utilized as the last high-risk criteria to strengthen the ability to identify patients with poor anticoagulation control on warfarin. The SAMe-TT2R2 was internally validated using data from the AFFIRM trial for patients with AF. ${ }^{[1]}$ Other external validations with real world patient cohorts have assessed the predictive value of this scoring tool. ${ }^{[17]}$ It has been associated with outcomes data, making it a potential first step in the patient management pathway to help in identifying patients at high-risk of poor anticoagulation control. Our study population included indications for VTE and NVAF. There are currently no validated warfarin risk scoring tools for VTE patients. Therefore, we utilized the SAMeTT2R2 score in our patient population because we consider clinical risk factors to be similar for both indications.

Initial appointments to transition to a DOAC required 3045 minutes compared to standard 15-minute warfarin clinic follow up appointments. The extended appointment times were needed for patient education, laboratory monitoring, and addressing financial barriers. However, DOAC follow up appointments via face-to-face or telephonic were easily manageable.

Adherence is an ongoing barrier to anticoagulation therapy. As a standard of care, warfarin patients in our anticoagulation clinic are provided adherence education with further adherence support such as pill boxes and calendars depending on patient barriers. Patients may continue to have poor adherence despite these interventions, potentially due to socioeconomic challenges. Of our entire clinic population, around $20 \%$ are uninsured and receive charity care. Furthermore, it is estimated that $50 \%$ of our clinic population is indigent, regardless of insurance status. Therefore, the monthly cost of warfarin, although priced at around $\$ 4$, is likely to be unaffordable for many patients, especially those

\section{REFERENCES}

[1] Pistoia F, Sacco S, Tiseo C, et al. The Epidemiology of Atrial Fibrillation and Stroke. Cardiol Clin. 2016 May; 34(2): 255-68. PMid: 27150174. https://doi.org/10.1016/j.ccl.2015.12.002

Published by Sciedu Press with numerous medications and in addition to copay costs for INR monitoring. Patients may also have transportation challenges understanding the necessity of treatment or may be apprehensive about adverse effects. Challenges following dosing instructions which can be complex with warfarin, remembering to take medications, and maintaining a consistent dietary intake may also be present in our population. Using the 3 criteria in this pilot study provided justification that the risk of clinical events outweighs the benefits of continuing or re-trying warfarin in the patients selected.

We utilized PDC, a method endorsed by the Pharmacy Quality Alliance to evaluate adherence by determining the proportion of days in the measurement period covered by prescription claims. A PDC threshold of $80 \%$ indicates the medication has a reasonable likelihood of achieving most of the potential clinical benefit. ${ }^{[18]}$ The high adherence rate for DOAC patients seen in this pilot study may be due to the repeated patient telephone outreach in which education and compliance was strongly emphasized in addition to reduced cost burden for many patients (e.g. free access to DOACs through PAPs, avoidance of office visit copays for warfarin INR monitoring). Other factors influencing patient adherence to DOAC therapy may be related to increased satisfaction over warfarin (see Table 3). ${ }^{[14]}$

After implementation of the warfarin-to-DOAC pilot, there was an increase in overall clinic TTR comparing pre- to postanalysis. Thus, although there is currently a lack of research on this concept, switching high-risk warfarin patients to other, potentially more suitable therapies, is a strategy for anticoagulation clinic improvement where TTR serves as a quality indicator. The initial lower TTR, and also appointment adherence, is potentially due to the aforementioned socioeconomic challenges in our patient population. These findings further support the importance of having a systematic approach for optimizing anticoagulation population management in the clinic setting.

\section{Conclunsions}

A pilot process was successful in reducing the percentage of high-risk warfarin patients by switching to DOAC therapy.

\section{CONFLicts OF INTEREST Disclosure}

The authors declare they have no conflict of interest.
[2] Colilla S, Crow A, Petkun W, et al. Estimates of current and future incidence and prevalence of atrial fibrillation in the U.S. adult population. Am J Cardiol. 2013 Oct 15; 112(8): 1142-7. PMid: 23831166. https://doi.org/10.1016/j.amjcard.2013.05.063 
[3] Spencer F, Emery C, Lessard D, et al. The Worcester Venous Thromboembolism study: a population-based study of the clinical epidemiology of venous thromboembolism. J Gen Intern Med. 2006; 21 : 722-727. PMid: 16808773. https://doi.org/10.1111/j.1525 $-1497.2006 .00458 . \mathrm{x}$

[4] Safavi-Naeini P, Saeed M. Target-specific oral anticoagulants: should we switch from warfarin? Tex Heart Inst J. 2015 Jun 1; 42(3): 229-33 PMid: 26175634. https : //doi .org/10.14503/THIJ-15-5065

[5] Ruff C, Giugliano R, Braunwald E, et al. Comparison of the efficacy and safety of new oral anticoagulants with warfarin in patients with atrial fibrillation: a meta-analysis of randomised trials. Lancet. 2014 Mar 15; 383(9921): 955-62. https ://doi.org/10.1016/S014 0-6736 (13) 62343-0

[6] January C, Wann L, Alpert JS, et al. 2014 AHA/ACC/HRS guideline for the management of patients with atrial fibrillation: a report of the American College of Cardiology/American Heart Association Task Force on Practice Guidelines and the Heart Rhythm Society. J Am Coll Cardiol. 2014 Dec 2; 64(21): e1-76. PMid: 24685669 https://doi.org/10.1016/j.jacc.2014.03.022

[7] Kearon C, Akl E, Ornelas J, et al. Antithrombotic Therapy for VTE Disease: CHEST Guideline and Expert Panel Report. Chest. 2016 Feb; 149(2): 315-52. PMid: 26867832. https://doi.org/10.1 $016 / \mathrm{j}$. chest . 2015.11 .026

[8] Heidbuchel H, Verhamme P, Alings M, et al. Updated European Heart Rhythm Association Practical Guide on the use of non-vitamin $\mathrm{K}$ antagonist anticoagulants in patients with non-valvular atrial fibrillation. Europace. 2015 Oct; 17(10): 1467-507. PMid: 26324838 https://doi.org/10.1093/europace/euv309

[9] Kazmi RS, Lwaleed BA. New anticoagulants: how to deal with treatment failure and bleeding complications. Br J Clin Pharmacol. 2011 Oct; 72(4): 593-603. PMid: 21752066. https://doi.org/10.1 $111 / j .1365-2125.2011 .04060 . x$

[10] Reiffel JA. Time in the therapeutic range for patients taking warfarin in clinical trials: Useful, but also misleading, misused, and overinterpreted. Circulation. 2017 Apr 18; 135(16): 1475-77. PMid: 28416519. https://doi.org/10.1161/CIRCULATIONAHA. 116 .026854
[11] Apostolakis S, Sullivan R, Olshansky B, et al. Factors Affecting Quality of Anticoagulation Control Among Patients With Atrial Fibrillation on Warfarin: The SAMe-TT 2 R 2 Score. Chest. 2013 Nov; 144(5): 1555-63. PMid: 23669885. https://doi.org/10.1378/ chest.13-0054

[12] Schulman S, Kearon C. Subcommittee on Control of Anticoagulation of the Scientific and Standardization Committee of the International Society on Thrombosis and Haemostasis. Definition of major bleeding in clinical investigations of antihemostatic medicinal products in non-surgical patients. J Thromb Haemost. 2005 Apr; 3(4): 6924. PMid: 15842354. https://doi.org/10.1111/j.1538-7836. 2005.01204.x

[13] Patel M, Mahaffey K, Garg J, et al. Rivaroxaban versus warfarin in nonvalvular atrial fibrillation. N Engl J Med. 2011 Sep 8; 365(10): 883-91. PMid: 21830957. https://doi.org/10.1056/NEJMoa 1009638

[14] Thompson AN, Ragucci KR, Fermo JD, et al. Evaluation of patient perceptions and outcomes related to anticoagulation point-of-care testing in ambulatory care clinics. Pharm Pract (Granada). 2009 Oct; 7(4): 213-7. https://doi .org/10.4321/S1886-36552009000 400004

[15] Brekelmans MP, Kappelhof M, Nieuwkerk PT, et al. Preference for direct oral anticoagulants in patients treated with vitamin $\mathrm{K}$ antagonists for venous thromboembolism. Neth J Med. 2017 Mar; 75(2): 50-55. PMid: 28276323.

[16] Jones D, Cook R, Rodriguez A, et al. Suggested running head: Personal HIV Knowledge, appointment adherence and HIV outcomes. AIDS Behav. 2013 January; 17(1): 242-249. PMid: 23143751. https://doi.org/10.1007/s10461-012-0367-y

[17] Esteve-Pastor M, Roldán V, Valdés M, et al. The SAMe-TT2R2 score and decision-making between a vitamin $\mathrm{K}$ antagonist or a non-vitamin $\mathrm{K}$ antagonist oral anticoagulant in patients with atrial fibrillation. Expert Rev Cardiovasc Ther. 2016; 14(2): 177-87. PMid: 26559964. https://doi.org/10.1586/14779072.2016.1116 941

[18] Nau D. Proportion of Days Covered (PDC) as a Preferred Method of Measuring Medication Adherence. Adherence. Accessed January 28, 2016. Available from: http://www.pqaalliance.org/imag es/uploads/files/pqapdcvsmpr.pdf 